\title{
Adaptive Control of Low-Frequency Acoustic Modes in Small Rooms
}

\author{
Sylvio R. Bistafa ${ }^{*}, 1$, Mark P. Hodgkin², William S. Morita ${ }^{2}$, Bruno O. Köhn² and João J. Neto \\ ${ }^{I}$ Department of Mechanical Engineering, Polytechnic School, University of São Paulo, 05508-900, São Paulo, SP, \\ Brazil \\ ${ }^{2}$ Department of Computing Engineering and Digital Systems, Polytechnic School, University of São Paulo, 05508-900, \\ São Paulo, SP, Brazil
}

\begin{abstract}
The work presents the implementation in software of an adaptive method for reducing the non-uniformity of the frequency-response-curve, by covering certain walls of small rectangular rooms, with a particular type of a passive acoustical treatment, consisting of porous absorbents covered with perforated sheets, in which each perforation behaves independently as Helmholtz resonators. The method employs new developments of another publication for finding the modal parameters, namely natural frequencies and associated damping constants, of rectangular rooms with arbitrary (uniform) wall-impedances. The adaptive software is capable of continuously improving the implemented type of acoustical treatment and would allow the implementation of new types of treatments as desired. Some testing results are presented, demonstrating the ability of the software for finding best parameters of the acoustical treatment, giving as input data the room dimensions and source/receiver locations only.
\end{abstract}

Keywords: Acoustics of small rooms, acoustic treatment of small rooms, control of low-frequency acoustic modes in small rooms, adaptivity applied to room acoustics.

\section{INTRODUCTION}

The rather large spacing of low-frequency acoustic modes in undamped small rooms, results in a frequencyresponse-curve with minima and maxima separated by several dBs. Depending on the placement of loudspeakers and listeners, the room dimensions, its surface conditions and contents, the resulting frequency-response-curve can become very uneven, which can compromise the acoustic quality of small critical listening rooms such as studios, home-theaters; communication rooms etc., because some frequencies will be overly accentuated, and others not adequately represented.

A room will be better if it had a more uniform frequencyresponse, which means a more uniform distribution of acoustic modes. Over the years, methods have been proposed to minimize the non-uniformity of frequency-responses of small rooms by redistributing the acoustic modes in the lowfrequency-range [1-4]. A review of the approaches in this special field of small room acoustics was given by Cox and D'Antonio [5]. All these methods have a serious limitation, since they are applicable only to rooms with rigid surfaces.

The calculation of the modal parameters of rectangular rooms with rigid surfaces (infinite wall-impedances) is rather straightforward [6]. The same calculation, whenever the room surfaces are hard (real and high wall-impedances) is more complex, but can still be done analytically [7].

*Address correspondence to this author at the Department of Mechanical Engineering, Polytechnic School, University of São Paulo, 05508-900, São Paulo, SP, Brazil; Tel: +55 (11) 3091-9888; Fax: +55 (11) 3813-1886;

E-mail: sbistafa@usp.br
One of the goals of the present work is to implement a new procedure to find the modal parameters of a rectangular room whenever its surfaces have arbitrary wall-impedances.

The determination of the modal parameters in the most general case of non-rectangular rooms requires one of the more complex methods, such as the use of the finite element method [8].

With the goal achieving a more uniform frequencyresponse, a dedicated finite element method has been proposed to redistribute the acoustic modes of a room in the low frequency range, which focused on several variable local geometric modifications of the room walls that form the independent variables of the optimization procedure [9].

Controlling sound reflections is another strategy used in critical listening rooms to avoid the variability of the frequency-response at the so-called 'sweet spot' [10]. This is a term used to describe the focal point between two speakers, where an individual is fully capable of hearing the stereo audio mix the way it was intended to be heard by the mixer. In the case of surround sound, this is the focal point between four or more speakers.

More often, passive techniques are used to reduce the variability of the frequency-response by means of 'basstraps', membrane absorbers and Helmholtz resonators.

The present work presents the implementation in software of an adaptive method for reducing the nonuniformity of the frequency-response-curve, at particular source/receiver locations, by covering certain walls of small rectangular rooms with a particular type of a passive acoustical treatment, consisting of Porous Absorbent 
Covered with Perforated Sheets (PAPS), in which each perforation behave independently as Helmholtz resonators.

The method employs new developments of another publication [12] for finding the modal parameters, namely natural frequencies and associated damping constants, of rectangular rooms with arbitrary (uniform) wall-impedances.

Adaptivity is the capacity of a system to take the decision to modify its own behavior in response to its history and input data, without the interference of an external agent. Adaptivity has been implemented in software capable of finding best parameters of the PAPS, giving as input data the room dimensions and source/receiver locations only. The adaptive software should be able to specify the characteristics of a PAPS acoustical treatment, and to suggest in which room-walls this treatment should be applied to.

\section{ACOUSTIC RESPONSE OF RECTANGULAR ROOMS WITH ARBITRARY (UNIFORM) WALL- IMPEDANCES}

Morse and Bolt [11] wrote the velocity potential for a standing wave in a rectangular room with dimensions $L_{x}, L_{y}$ and $L_{z}$ as

$\psi(\omega ; x, y, z)=D(x) \cdot E(y) \cdot F(z) \cdot e^{i \omega t}$,

where $\omega$ is the driving angular frequency and

$D(x)=\cosh \left[\left(\pi i x / L_{x}\right) \chi_{x}-\varphi_{x}\right]$,

with similar expressions for $E$ and $F$. The complex wave number in the x-direction is $\pi \chi_{x} / L_{x}$; and $\chi_{x}$, in terms of its real and imaginary parts can be written as $\chi_{x}(\omega)=\mu_{x}+i \kappa_{x}$, where $\mu_{x}$ is called the wave number parameter and $\kappa_{x}$ is called the attenuation parameter.

In the case of damped standing waves, the wave number ends up being generalized to a complex quantity whose imaginary part is the spatial attenuation factor given by $\pi \kappa_{x} / L_{x}$.

Here $\chi_{x}$ are the roots of the following equation [3]

$\pi i \chi_{x}+\operatorname{coth}^{-1}\left[\left(\frac{\zeta_{x_{1}}}{\eta_{x}}\right) \chi_{x}\right]+\operatorname{coth}^{-1}\left[\left(\frac{\zeta_{x_{2}}}{\eta_{x}}\right) \chi_{x}\right]=0$,

with two other equations for $\chi_{y}$ and $\chi_{z}$.

In Eq. (3), $\zeta_{x_{1}}$ and $\zeta_{x_{2}}$ are the specific impedances for the $x$-walls, and $\eta_{x}=\left(\omega L_{x} / \pi c\right)$, is called the frequency parameter, and gives the $x$-dimension of the room in half wavelengths.

Eq. (3) has an infinite number of roots $\chi$. The different roots are distinguished by assigning to them different values of the subscript $n$, with $n=0$ for the value of $\chi$ with the smallest value of $\mu, n=1$ for the next smallest value of $\mu$, and so on.

Bistafa and Morrissey [12] developed two numerical procedures for finding the roots of Eq. (3), for room-walls with arbitrary (uniform) wall-impedances. One of these procedures applies Newton's method and the other the homotopic continuation procedure.

Morse and Bolt [11] have shown that the stationary complex sound pressure at position $x, y, z$ in the room is given by $\hat{p}(x, y, z)=-i \rho \omega \Psi$, where $\rho$ is the density of air and $\Psi$ is given by

$\Psi=-c^{2} \sum_{n} \frac{B_{n} \psi_{n}(-\omega ; x, y, z)}{\omega^{2}-\left(\omega_{n}-i k_{n}\right)^{2}} e^{-i \omega t}$.

In Eq. (4) $B_{n}$ is given by

$B_{n}=\frac{Q_{0} \psi_{n}\left(-\omega ; x_{0}, y_{0}, z_{0}\right)}{\Delta_{n}(-\omega)}$,

where $Q_{0}$ is the strength of the source located at $x_{0}, y_{0}, z_{0}$, and the normalization factor $\Delta_{n}$ is given by

$\Delta_{n}(-\omega)=\frac{L}{2}\left\{1+\frac{i \beta_{1}(\eta / \pi)}{\chi_{n}^{2}(\omega)-\beta_{1}^{2} \eta^{2}}+\frac{i \beta_{2}(\eta / \pi)}{\chi_{n}^{2}(\omega)-\beta_{2}^{2} \eta^{2}}\right\}$,

where $\beta_{1}$ and $\beta_{2}$ are the admittances of two parallel walls $(\beta=1 / \zeta)$.

In Eq. (4) $\omega_{n}$ is the natural angular frequency of the $n^{\text {th }}$ mode given by

$\omega_{n}(\omega)=\pi c\left[\frac{\mu_{x}^{2}-\kappa_{x}^{2}}{L_{x}^{2}}+\frac{\mu_{y}^{2}-\kappa_{y}^{2}}{L_{y}^{2}}+\frac{\mu_{z}^{2}-\kappa_{z}^{2}}{L_{z}^{2}}\right]^{1 / 2}$,

and $k_{n}$ is the damping constant of the $n^{\text {th }}$ mode given by

$k_{n}(\omega)=\pi c\left[\frac{\mu_{x} \kappa_{x}}{\eta_{x} L_{x}}+\frac{\mu_{y} \kappa_{y}}{\eta_{y} L_{y}}+\frac{\mu_{z} \kappa_{z}}{\eta_{z} L_{z}}\right]$,

where $c$ is the sound velocity.

Newton's method was employed to find the roots of Eq. (3), which enabled the calculation of the natural angular frequencies and the damping constants of the room-modes by Eq. (7) and Eq. (8), respectively, and the stationary sound pressure for each driving frequency $f=\omega / 2 \pi$, by the equations given above, which allow the construction of the frequency-response-curve.

\section{THE IMPEDANCE OF POROUS ABSORBENTS COVERED WITH PERFORATED SHEETS}

Information regarding the specific impedances of the room-walls is necessary in order to apply the method presented in Section 2 to find the frequency-response-curve. The PAPS was the acoustical treatment chosen to control the low-frequency acoustic modes in small rectangular rooms. 


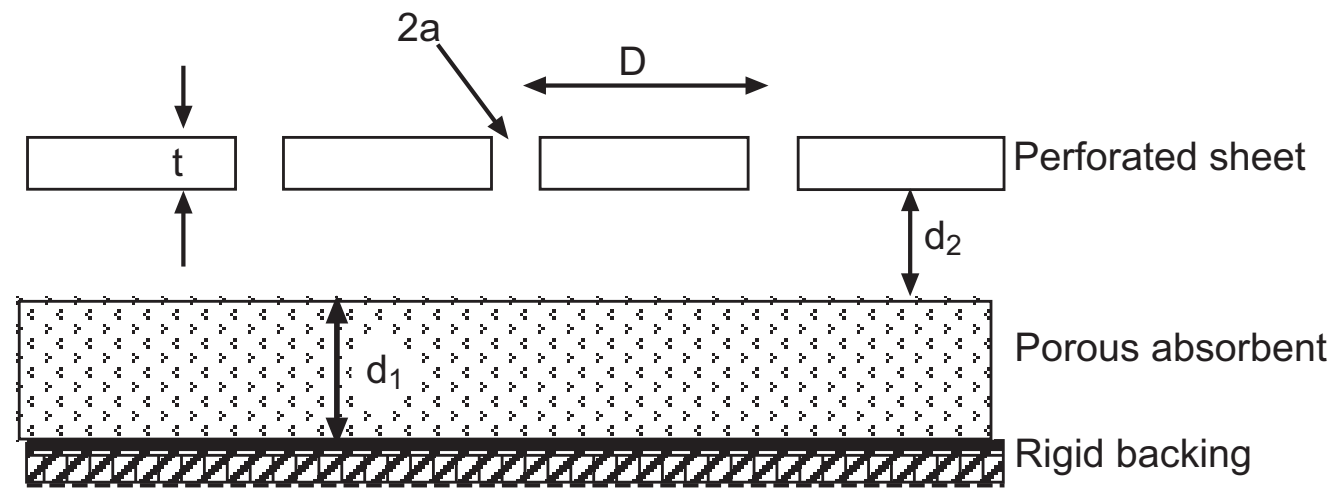

Fig. (1). Schematic of a porous absorbent covered with a perforated sheet (PAPS).

Fig. (1) shows the schematic of a porous absorbent covered with a perforated sheet.

According to ref. [13], the impedance at the top of the porous absorbent, $z_{1}$, is given by

$z_{1}=-i z_{i} \cot \left(k_{i} d_{1}\right)$

where $z_{i}$ is the characteristic impedance and $k_{i}$ the wave number of the porous absorbent.

The impedance at the top of the air layer and just below the perforation, $z_{2}$, is given by

$z_{2}=\frac{-z_{1} i \rho c \cot \left(k d_{2}\right)+\rho^{2} c^{2}}{z_{1}-i \rho c \cot \left(k d_{2}\right)}$,

with $k=\omega / c$.

Finally, the impedance at the top of the perforated sheet, $z_{3}$, is found by modeling the perforated surface as individual cells, which are assumed to behave independently as Helmholtz resonators, with a repeat distance D. This impedance is given by

$z_{3}=\frac{\rho}{\varepsilon}\left(\frac{t}{2 a}+1\right) \sqrt{8 v \omega}+(2 \delta a+t) \frac{i \omega \rho}{\varepsilon}+z_{2}$,

where $\varepsilon=\pi a^{2} / D^{2}, v$ is the kinematic viscosity of air, $\delta$ is a correction factor given by $\delta=0,8\left(1-1,4 \varepsilon^{1 / 2}\right)$, and $t$ is the thickness of the perforated sheet.

\section{THE INTEGRATION OF ADAPTIVE SOFTWARE WITH ROOM ACOUSTICS}

This Section presents how the adaptive concept was implemented in software to improve the frequency-responsecurve of small rooms at low-frequencies.

\subsection{Adaptivity}

Adapt means behavior change depending on circumstances. In control systems, an adaptive control is one that can modify the system behavior in response to changes in the dynamics of the process in face of disturbances.

Adaptivity can also be considered as the capacity of a system to take the decision to modify its own behavior in response to its history and input data, without the interference of an external agent. Adaptivity differs from Optimization because two identical instances of an adaptive system can evolve to complete different states, according to the diversity of events to which its operation has been submitted.

A decision tree is a graphical tool which allows the representation of the decisions taken by the system, the possible options and paths to be followed, the consequences of each decision, and the resulting final state. As such, the vertices of a decision tree represent the tests to be performed, and the branches represent the possible results.

Suppose the following sets: $\mathrm{N}=\{0,1,2\}, \mathrm{L}=\{\mathrm{a}, \mathrm{b}\}$, $\mathrm{C}=\{$ yes, no $\}$; and the function $\mathrm{F}: \mathrm{N} \times \mathrm{L}=\mathrm{C}=\{((0, \mathrm{a}), \mathrm{yes})$, $((0, b)$, yes $),((1, a)$, no $),((1, b)$, no $),((2, a)$, yes $),((2, b)$, no $)\}$. This function can be represented by any of the two trees shown in Fig. (2).

Fig. (2) shows conventional decision trees - nonadaptive. A decision tree can have extended functionalities for the inclusion of the adaptive process, to allow the creation of new paths or the modification of existing ones according to its use.

Fig. (3) shows the evolution of a decision tree as it receives new inputs.

\subsection{Cost Parameter}

The first step in the development of the software intelligence is the creation of a 'cost parameter'. On the assumption that the best acoustical treatment is the one which produces the flattest frequency-response-curve, then the cost parameter should be a measure of how much a given frequency-response-curve deviates from a flat-response.

The cost parameter adopted in the present study, is given by the sum of three grades given to the frequency-responsecurve.

The first grade is the standard deviation $\varepsilon$ of the frequency-response-curve from the best fit straight line derived from such a curve, which is given by

$\varepsilon=\sqrt{\frac{\sum_{n=1}^{N}\left(L_{p, n}-m f_{n}+c\right)}{N-1},}$ 

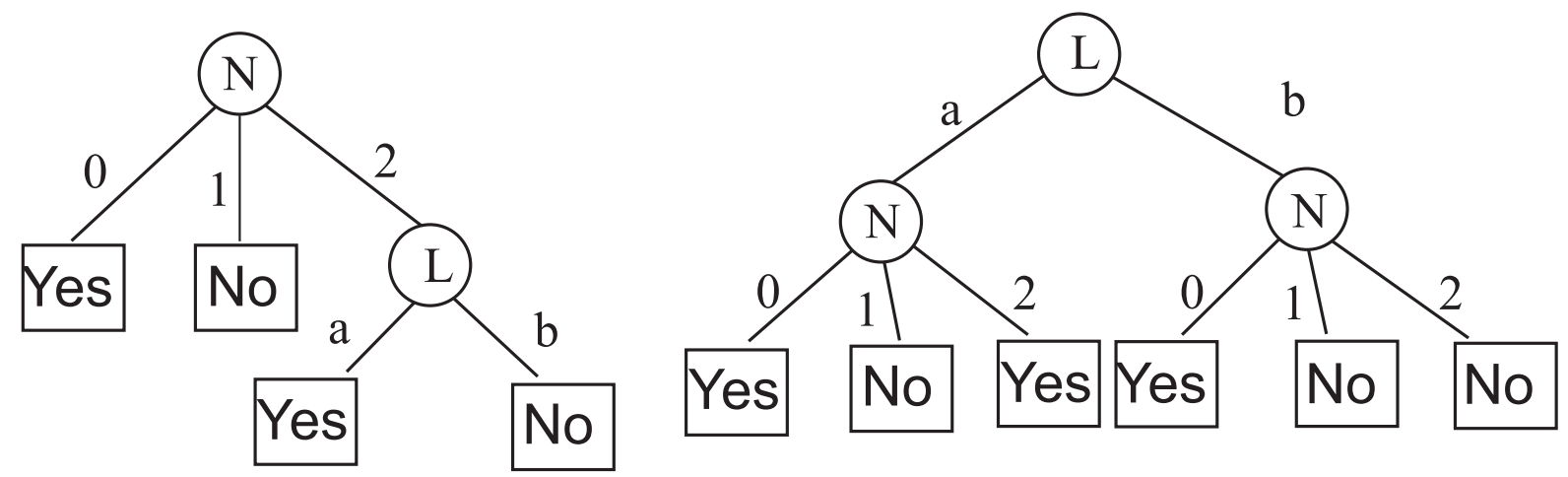

Fig. (2). Two decision trees for the same function $F$ : $N \times L=>C$.
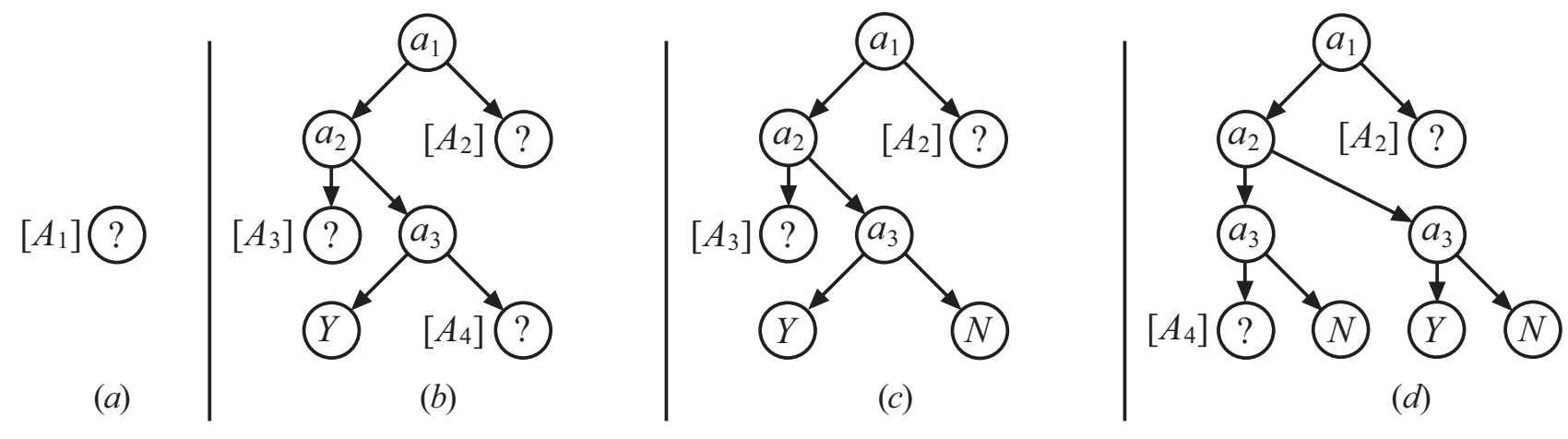

Fig. (3). The evolution of an adaptive decision tree.

where $L_{p, n}$ is the sound pressure at frequency $f_{n}, m$ is the slope, $\mathrm{c}$ is the intercept of the best fit line, and $N$ is the number of discrete frequencies where the sum is taken.

The second grade is the sum at $N$ discrete frequencies of the absolute value of the differences between the frequencyresponse-curve and a horizontal line at $0 \mathrm{~dB}$ (flat frequencyresponse).

The third grade is the population of peaks, which is formed by subdividing the frequency-response-curve in 10 , 8,6 , and 4 frequency-bands of the same width. In each band, the differences between the maximum and minimum are summed.

\subsection{Solutions for the Treatment of the Acoustic Modes}

Solutions for the treatment of the acoustic modes will be developed in two main steps as follows.

\subsubsection{Identification of which Decision Table to Use}

The software provides four decision tables, which allows the choice of which walls of the room should be treated: one wall, two walls, four walls, and five walls (it has been decided not to treat the floor). It has been found that the best combinations were those for which the two opposing walls had the same treatment.

\subsubsection{Identification of the Best PAPS}

Identification of the best PAPS found so far, iterate its dimensions, based on the method presented in Section 2, and to save the best PAPS for the case being considered. The PAPS parameters were allowed to vary in the following ranges: $d_{2}: 0,01 \mathrm{~m}-0,05 \mathrm{~m}, D: 0,005 \mathrm{~m}-0,01 \mathrm{~m}, t:$ $0,001 \mathrm{~m}-0,007 \mathrm{~m}, a: 0,0005 \mathrm{~m}-0,005 \mathrm{~m}, d_{1}: 0,0381 \mathrm{~m}$ or $0,0635 \mathrm{~m}$ or $0,0889 \mathrm{~m}$. The values of the thickness $d_{1}$ were chosen for the porous absorbent for which the impedance at its top $z_{1}$ was known from published results [13].

Two situations are possible. First situation: the room has been analyzed in the past; the previous PAPS is the starting point of new iterations, given an opportunity for the system to find a better solution, after the program has run for a certain period of time. Second situation: the room has never being analyzed so far; a search for rooms with similar characteristics is initiated, returning the PAPS which gives the best result for this new room. With this initial approximation, the system follows the first situation above.

Besides performing iterations with the PAPS parameters, the software is capable of random searches as well.

For the iterations, the software searches for results already existent in the data base, that is, results from 'previous experiences'. From these, a 'calibration' of the parameters is performed with the goal of finding a better solution.

The random searches are performed to widen the options of possible solutions, in order to avoid the concentration of efforts in one direction only. A random search is performed after three iterations, with the values of the parameters previously obtained. As new solutions are found (the best solutions); they are saved. In this way, the data base always increases. Fig. (4) presents the adaptive software flow diagram. 


\section{SIMULATION RESULTS AND DISCUSSION}

Simulations were performed to check the functionality of the adaptive software. For these simulations, the source was located at one of the corners of the room - to excite all possible acoustic modes — , and the receiver was positioned at the center of the room.

The software initially assumes that the specific impedance of the walls of the bare room is real and has the

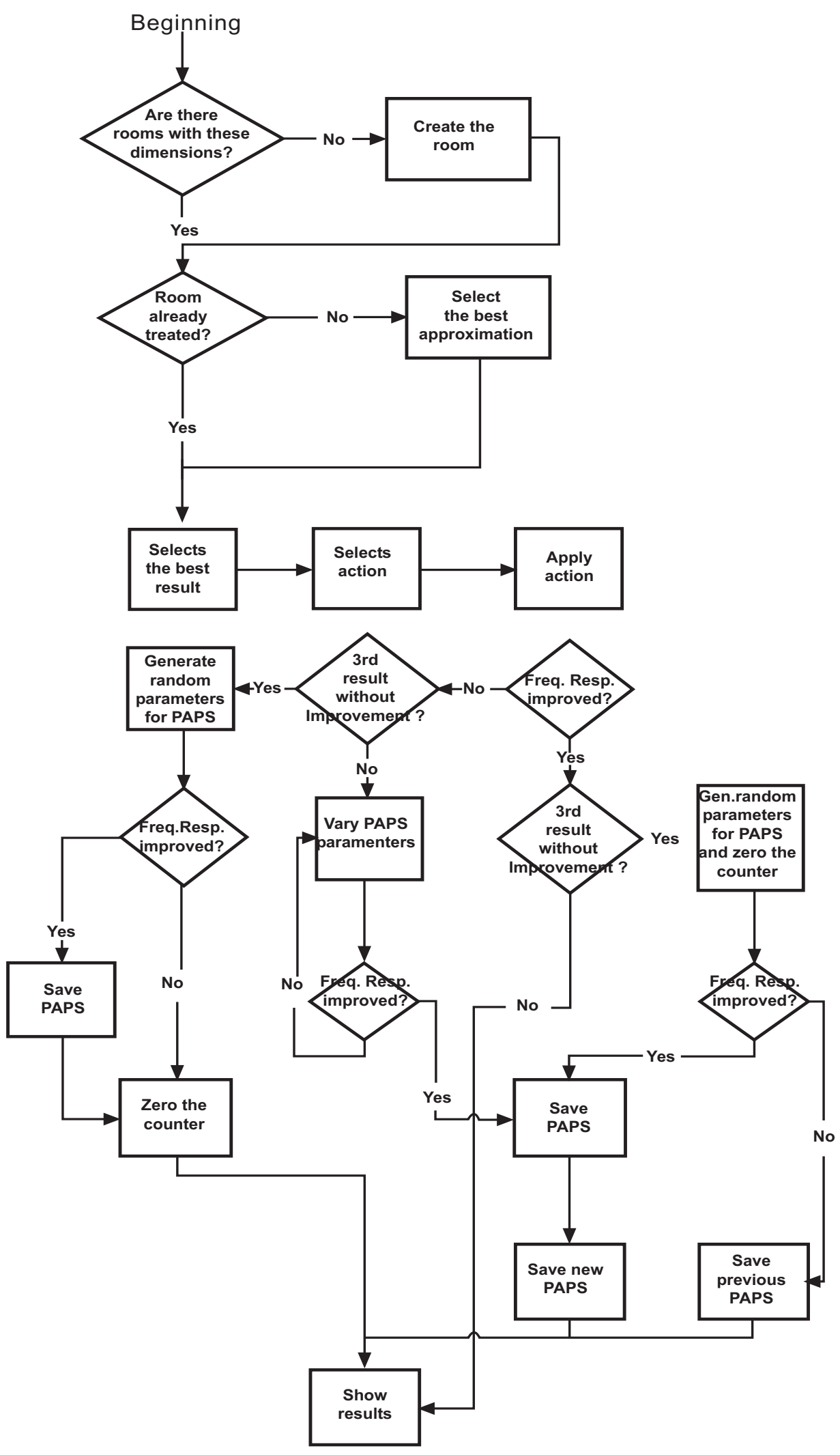

Fig. (4). Adaptive software flow diagram. 
value 300 (hard walls) [12]. Essentially, by varying the dimensions o PAPS, namely, $d_{1}, d_{2}, D, a$, and $t$, different frequency-response-curves can be obtained.

Fig. (5) shows three frequency-response-curves of a $3 \mathrm{~m}$ x $3 \mathrm{~m}$ x $3 \mathrm{~m}$ room without treatment (bare room), and two solutions generated by the adaptive software: an intermediate treatment, and the final treatment applied to the whole four verticals walls of the room, with the PAPS having the following dimensions: $d_{2}=0,01 \mathrm{~m}, \quad D=0,045 \mathrm{~m}$, $t=0,001 \mathrm{~m}, a=0,005 \mathrm{~m}, d_{1}=0,0635 \mathrm{~m}$. dimensions: $d_{2}=0,01 \mathrm{~m}, \quad D=0,035 \mathrm{~m}, \quad t=0,004 \mathrm{~m}$, $a=0,005 \mathrm{~m}, d_{1}=0,0635 \mathrm{~m}$.

It can be seen in Figs. $(\mathbf{5 , 6})$ that the acoustical treatments generated by the adaptive software reduced considerably the irregularities of the frequency-response-curves, which have produced, for these rooms, reductions of up to $15 \mathrm{~dB}$ at certain frequencies, and an almost flat frequency-response for frequencies above $100 \mathrm{~Hz}$.

Practical critical issues related to the successful implementation of the PAPS and other types of acoustical

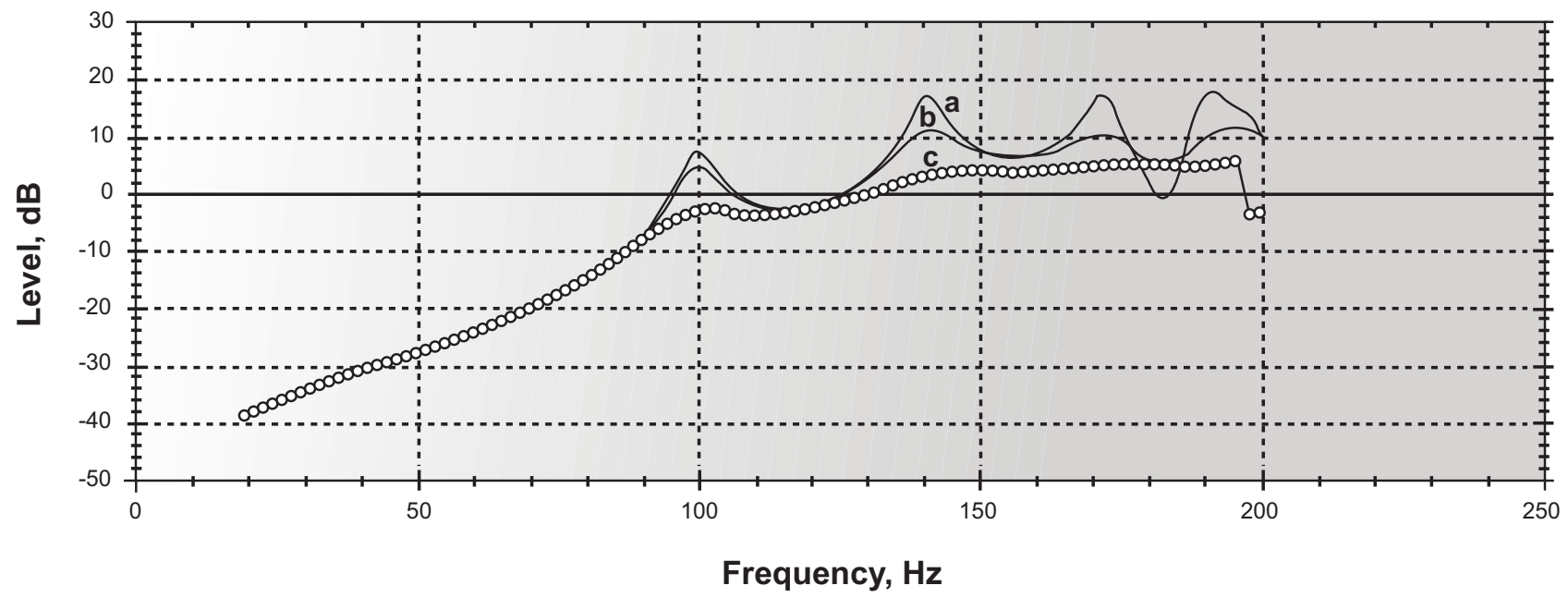

Fig. (5). Frequency-response-curves of a $3 \mathrm{~m} \mathrm{x} 3 \mathrm{~m} \times 3 \mathrm{~m}$ room: a) without treatment (bare room), b) an intermediate treatment, c) final treatment applied to the whole four verticals walls of the room, with the PAPS having the following dimensions: $d_{2}=0,01 \mathrm{~m}, D=0,045 \mathrm{~m}$, $t=0,001 m, a=0,005 m, d_{1}=0,0635 m$.

Fig. (6) shows two frequency-response-curves of a $4 \mathrm{~m} \mathrm{x}$ $3 \mathrm{~m} \times 2,5 \mathrm{~m}$ room without treatment (bare room), and the final solution generated by the adaptive software, which resulted in a treatment applied to the whole four vertical walls of the room, with the PAPS having the following treatments using the procedures here presented are, in the case of the PAPS, the availability of data related to the impedance $z_{1}$ at the top of the porous absorbent; for the other types of acoustical treatments, the knowledge of analytical expressions for the impedance of the proposed treatment.

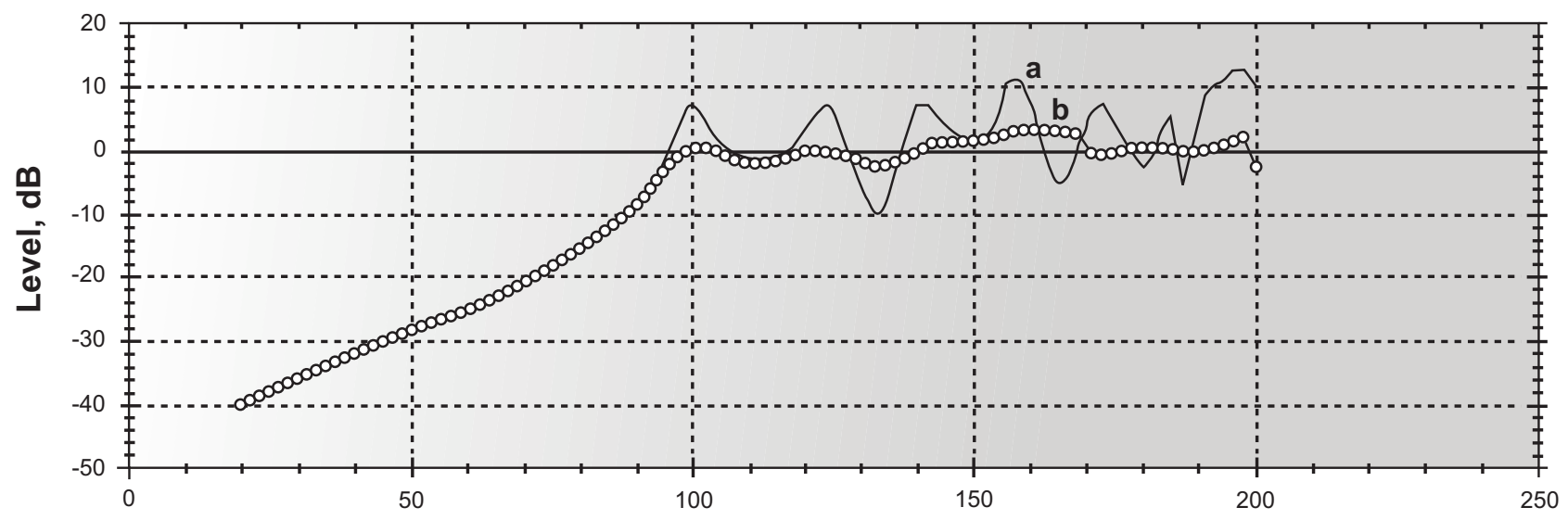

Frequency, $\mathrm{Hz}$

Fig. (6). Frequency-response-curves of a $4 \mathrm{~m} \times 3 \mathrm{~m} \times 2,5 \mathrm{~m}$ room: a) without treatment (bare room), b) treatment applied to the whole four vertical walls of the room, with the PAPS having the following dimensions: $d_{2}=0,01 \mathrm{~m}, D=0,035 \mathrm{~m}, t=0,004 \mathrm{~m}, a=0,005 \mathrm{~m}$, $d_{1}=0,0635 \mathrm{~m}$. 


\section{CONCLUSIONS}

Adaptive software has been developed that allows selection of the characteristics of porous absorbents covered with perforated sheets for covering the walls of small rectangular-rooms in order to reduce the non-uniformity of the frequency-response-curve. The software implements new developments of another publication for finding the modal parameters, namely natural frequencies and associated damping constants of rectangular rooms with arbitrary (uniform) wall-impedances. As such, the adaptive software is capable of continuously improving the implemented type of acoustical treatment and allows the implementation of new treatments as desired.

Some results have been presented, demonstrating the ability of the software for finding best parameters of an acoustical treatment based on porous absorbents covered with perforated sheets.

\section{ACKNOWLEDGEMENT}

Declared none.

\section{CONFLICT OF INTEREST}

Declared none.

\section{REFERENCES}

[1] Bolt RH. Note on the normal frequency statistics in rectangular rooms. J Acoust Soc Am 1946; 18(1): 130-3.

[2] Louden MM. Dimension ratios of rectangular rooms with good distribution of eigentones. Acustica 1971; 24: 101-4.

[3] Gilford CLS. The acoustic design of talk studios and listening rooms. J Audio Eng Soc 1979; 27: 17-31.

[4] Bonello OJ. A new criterion for the distribution of normal room modes. J Audio Eng Soc 1981; 29(9): 597-605.

[5] Cox TJ, D'Antonio P, Avis MR. Room sizing and optimization at low frequencies. J Audio Eng Soc 2004; 52(6): 640-51.

[6] Jones D. Small Room Acoustics. In: Ballou GL Ed. Handbook for Sound Engineers. Massachusetts: Focal Press 2005; pp. 89-107.

[7] Kinsler LE, Frey AR, Coppens AB, Sanders JV. Fundamentals of Acoustics. New York: John Wiley \& Sons, Inc 2000.

[8] van Nieuwland JM, Weber C. Eigenmodes in Non-Rectangular Reverberation Rooms. Noise Control Eng 1979; 13(3): 112-21.

[9] Papadopoulos CI. Redistribution of the low frequency acoustic modes of a room: a finite element-based optimization method. Appl Acoust 2001; 62: 1267-85.

[10] Walker R. A Controlled-Reflection Listening Room for Multichannel Sound. Proc Inst Acoust 1998; 209(5): 25-36.

[11] Morse PM, Bolt RH. Sound waves in rooms. Rev Mod Phys 1944; 16: $69-150$.

[12] Bistafa SR, Morrissey JW. Numerical solutions of the acoustic eigenvalue equation in the rectangular room with arbitrary (uniform) wall impedances. J Sound Vib 2003; 263: 205-18.

[13] Cox TJ, D'Antonio P. Acoustic Absorbers and Diffusers. London: Spon Press 2004. 